\title{
Optimization Model of Wind Farm
}

\author{
Xueying Ding
}

\section{North China Electric Power University , Baoding 071000 , China} 1476848927@qq.com

\begin{abstract}
For the optimization of wind farm layout, the model of fan wake model, fan cost benefit model and wind farm incremental capacity evaluation model are established in this paper. The simulation of the wake effect of the wind turbine is the basis for the prediction of the generation efficiency of the wind farm. The cost-benefit model of the wind farm is used to calculate the cost of the wind turbine with the installed cost of the fan. The wind farm incremental capacity evaluation model is used to analyze the comparative evaluation of the selected different fan arrangement. And finally determine the optimal arrangement of the fan for a given area wind farm.
\end{abstract}

Keywords: wind farm optimization

farm wake model cost-benefit model wind farm incremental capacity evaluation model Niche Genetic Algorithm

\section{Introduction}

Wind pow uses wind power to promote wind turbine blade rotation, and then increase the speed of rotation through the speed-up machine to promote power generation. According to the current windmill technology, they can generate electricity at about $3 \mathrm{~m} / \mathrm{s}$ of the wind speed (the degree of breeze). Now wind power is forming a boom in the world, because wind power is no fuel problem, and it will not produce radiation or air pollution. Wind power in Finland, Denmark and other countries is very popular, and in the western China it is also strongly advocated.

\section{Model establishment}

In optimizing the fan arrangement, three elements should be consider:(1) the distribution of wind resources; (2) the interaction between the wind turbine, that is, the wake effect; (3) the optimized wind farm should provide more power, and at the same time ensure the lowest cost of wind farm construction. Aiming at the problem of wind farm optimization,the following models is established in this paper to study the optimization of domestic wind farm layout[1].

\subsection{Wake model}

Based on the estimation of the efficiency of the wind farm and the correctness of the optimal arrangement of the fan, the fan wake model is established. The simulation of the wake effect of the fan is the basis for the prediction of the power generation efficiency of the whole wind farm.

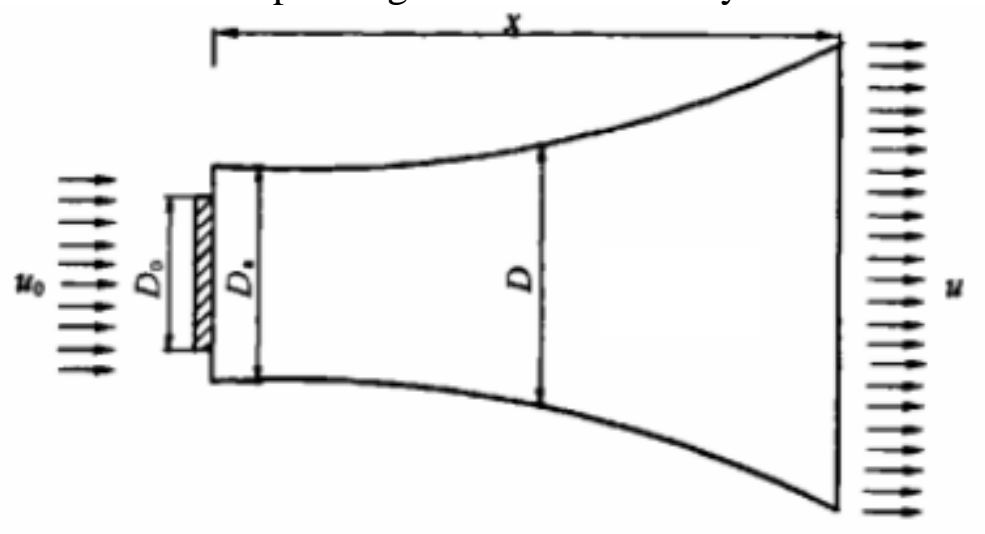

Figure1.control system 
As shown in the figure1, the control volume method is used for wind turbine flow analysis. $u_{0}$, $u$ are the wind speeds before and after the wind wheel at the distance from the wind wheel $\mathrm{x}$ meters, $D_{0}$ is wind wheel diameter; $D_{a}$ 、D are wake diameters before and after the wind wheel at the distance from the wind wheel $\mathrm{x}$ meters.The wake flow after the wind wheel at the distance from the wind wheel $x$ meters is chosen as the control body.

Assuming that the radius of the fan wake increases linearly with distance,so

$$
\begin{aligned}
& D(x)=D_{a}\left[1+2 k\left(x / D_{a}\right)\right] \\
& D_{a}=D_{0} \sqrt{(2-a) / 2(1-a)}
\end{aligned}
$$

Where $\mathrm{k}$ is the constant that represents the angle of the influence area of the fan wake, and it can be given by the following empirical formula[2]:

$$
k=\frac{0.5}{\ln \left(Z / r_{e}\right)}
$$

Where, $\mathrm{Z}$ refers to the fan center height of the horizontal axis, $r_{e}$ refers to the surface roughness.

$a$ is the rate of decay after the wind passes through the fan, i.e

$$
a=1-u_{a} / u_{0}
$$

the calculation of the affected area of fan wake, is divided into the following two cases according to the wind direction.

(1) Straight wind direction: including the wind direction of $0^{\circ} 、 90^{\circ} 、 180^{\circ} 、 270^{\circ}$. If the spacing between the grid center points is greater than the maximum wake radius of the fan, the fan wake does not affect both sides of the fan. At this time the distance between the upstream and downstream fans of the impact of the wake is:

$$
x=d_{x y}=\sqrt{\left(x_{i}-x_{k}\right)^{2}+\left(y_{i}-y_{p}\right)^{2}}
$$

(2) Oblique wind direction: any other direction in addition to direct wind. At this point we must judge whether the downstream fan is in the affected area of the upstream fan,as shown in figure 2. For fan placement points $\left(x_{k}, y_{p}\right),\left(x_{i}, y_{i}\right)$, if satisfied

$$
\left[\tan (\beta+\arctan \alpha)\left(x_{k}-x_{i}\right)+y_{i}\right] \leq y_{p} \leq\left[\tan (\beta-\arctan \alpha)\left(x_{k}-x_{i}\right)+y_{i}\right]
$$

it is stated that the placement point $\left(x_{k}, y_{p}\right)$ is within the wake influence area of the upstream distribution point $\left(x_{i}, y_{i}\right)$. At this point, the wake distance between the upstream and downstream fans is

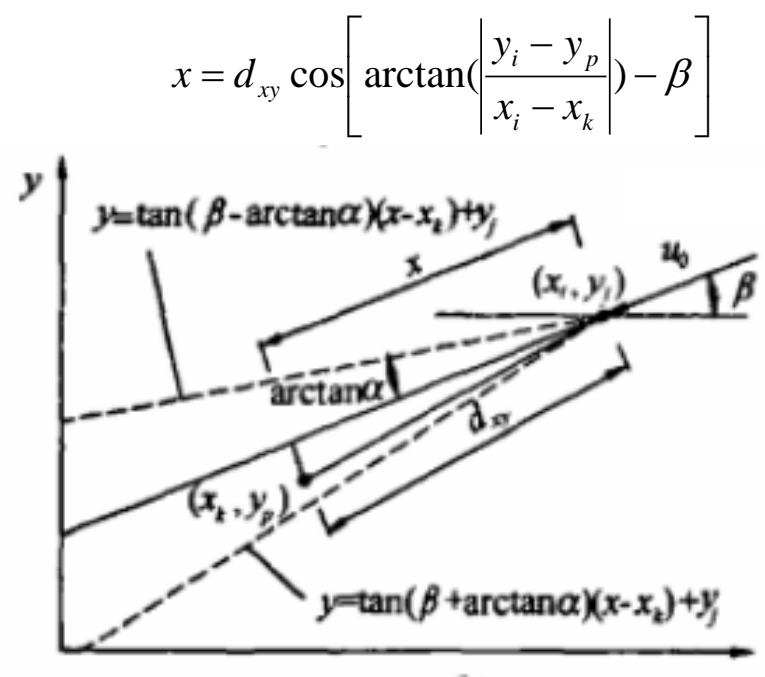

Figure2.A diagram of the affected area of the wake 


\subsection{Cost-benefit model}

Considering a selected fan layout scheme and related investment cost, the cost-benefit model of the wind farm fan is established, and then the cost of the power generation in the evaluation period is estimated combining with the wake model. The rationality of the model will be directly affects the economic evaluation conclusion of fan arrangement.

In the optimal layout of the fan, only need to consider the total cost of investment with the wind turbine equipment $C_{\text {total }}$, including the fixed investment costs associated with the fan equipment $C_{1}$ and the total operating maintenance and management costs during the evaluation period $C_{2} . C_{1}$ is estimated by the cost of wind farm related equipment, and $C_{2}$ is relative with labor costs, management fees and unit overhaul costs and so on. In order to simplify the calculation, the annual operating maintenance costs are expressed as $\alpha C_{1}$, the proportional function of $C_{1}[2,3]$ :

$$
C_{\text {total }}=C_{1}+C_{2}=C \cdot N\left(2+e^{-0.00174 N^{2}}\right) /[3 \cdot(1+T \cdot \alpha)]
$$

Where $C$ represents the converting cost of unit wind turbine, $T$ represents the cycling time of wind farm benefit evaluation, $\mathrm{N}$ represents the total number of wind farm fans, $\alpha$ represents the ratio of the total cost of operation per unit and maintenance of the unit per year to $C_{1}$.

On the wind farm benefits, the power generation revenue is generally calculated. In the selected wind farm assessment period, the total output of the wind farm fan $P_{\text {total }}$ can be calculated according to the average wind speed probability density function $P E(u)[4]$ and fan-power curve $P(v) \sim v[5]$ :

$$
P_{\text {total }}=\int_{0}^{\infty} P E(u)\left[\sum_{i=1}^{N} P\left(v_{i}\right) d u\right]
$$

Where, $u$ represents the average natural wind speed in wind farm, $v$ is the wind speed of the fan under the influence of the wake effect.

\section{3the evaluation model of incremental capacity of wind farm}

Considering the comparative evaluation and analysis of the selected different fan arrangement, the evaluation model of incremental capacity of wind farm is established.

The ultimate goal of the wind turbine layout is to optimize the layout, making the ratio of total input to total output of wind farms minimize, that is, the benefit of unit investment is maximize. The evaluation objective function is

$$
\min \left(C_{\text {total }}^{\prime} / P_{\text {total }}\right)
$$

We use the incremental evaluation method to evaluate the cost-effectiveness ratio of increased fan in the farm, that is, at the same conditions, such as terrain, wind energy and fan conditions, increase $(\mathrm{N}+1)^{\text {th }}$ fan on the basis of $\mathrm{N}$ fans, then compare the input of the wind farm with the increment of the output under two conditions. If

$$
I_{n c r}=\Delta C_{\text {total }}^{\prime} / \Delta P_{\text {total }} \geq\left(C_{\text {total }}^{\prime} / P_{\text {total }}\right)_{N}
$$

it shows that the economy of the farm is worse after increasing $(\mathrm{N}+1)$ th fan. So the wind farm in the layout of the $\mathrm{N}$ fans is the best.

\section{Model solving}

The specific calculation process is as follows:

1. Divide the wind farm into a square grid of $m^{*} n$, assuming that the fan is only arranged at the center of each grid and the grid side is longer than the maximum wake radius produced by the fan. Each of the arrangements for the wind farm fan is treated as an individual and a grid point with a fan is set to " 1 " and the other points are " 0 ".

2. Select " $n$ " as the size of the initial population. Each individual has $N$ grid points value of 1 , that is, the wind farm places $\mathrm{N}$ fans, and the randomly give $\mathrm{n}$ fan arrangements in wind farm.

3.According to the above-mentioned cost-benefit model, the fitness function of the genetic algorithm is established and calculated fitness $(N)=C_{\text {total }}^{\prime} / P_{\text {total }} . \mathrm{N}$ individuals with smaller fitness 
values are selected to enter the mating pool $i$ as the parents to produce (n-1) progeny, and make up new populations with the individuals of best fit values . It should be noted that, in order to ensure that the number of fans unchanged in the process of individual crossings, the algorithm selects $\mathrm{k}$ genes from a parent twice in the random, and two selected genes are different, then the two selected genes cross New individual.

4. Set the maximum evolutionary algebraic control value for the optimal layout of the wind farm fan generation $=1000$. If the evolutionary algebra exceeds this control value, the program is executed and the result is output. The evaluation model of the incremental capacity of the wind farm is selected as the termination condition of the calculation.

\section{Conclusions}

On the basis of the relevant research achievements abroad, the model of fan wake model, fan cost - benefit model, wind farm incremental capacity evaluation model are established, and then the wind turbine fan layout is evaluated from three different aspects, finally the optimal arrangement of the fan for a given area wind farm is determined. In this way, we can make more efficient use of wind energy resources to improve the economy of wind farms.

\section{References}

[1]Tian Linlin, Zhao Ning, Zhong Wei, Placement Optimization of Wind Farm Based on Niche Genetic Algorithm[J], Journal of Nanjing University of Aeronautics\&Astronautics.(2011)

[2] Mosstti G, Poloni C, Diviacco B, Optimization of wind turbine positioning in large wind farms by means of a genetic algorithm[J]. Journal of Wind Engineering and Industrial Aerodynamics.

[3] Grady S A, Hussaini M Y, Abdullah M M. Placement of wind turbines using genetic algorithms[J] Renewable energy,2005(30)

[4] Ming Ding, Yichun Wu, Lijun Zhang, Study on Wind Probability of Wind Farm[J], Journal of China Electromechanical Engineering, 2005

[5]Deyou Liu, Zhizhong Tan, Feng Wang, Simulation Study on Joint Operation System of Wind Power - Pumping Storage[J],Hydropower and Energy Science,2006 